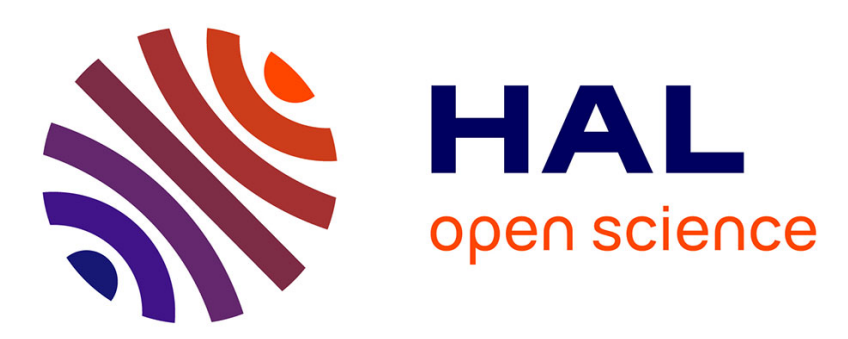

\title{
Residue phase optimization for power oscillations damping control revisited
}

Bogdan Marinescu

\section{To cite this version:}

Bogdan Marinescu. Residue phase optimization for power oscillations damping control revisited. Electric Power Systems Research, 2019, 168, pp.200-209. 10.1016/j.epsr.2018.11.007 . hal-02510481

\section{HAL Id: hal-02510481 \\ https://hal.science/hal-02510481}

Submitted on 17 Mar 2020

HAL is a multi-disciplinary open access archive for the deposit and dissemination of scientific research documents, whether they are published or not. The documents may come from teaching and research institutions in France or abroad, or from public or private research centers.
L'archive ouverte pluridisciplinaire HAL, est destinée au dépôt et à la diffusion de documents scientifiques de niveau recherche, publiés ou non, émanant des établissements d'enseignement et de recherche français ou étrangers, des laboratoires publics ou privés. 


\title{
Residue Phase Optimization for Power Oscillations Damping Control Revisited
}

\author{
Bogdan Marinescu \\ Ecole Centrale de Nantes - LS2N, 1 Rue de la Noë, 44321, Nantes, France
}

\begin{abstract}
One of the most used way to tune the parameters of power oscillations damping controllers (like, e.g., the Power System Stabilizers (PSS) of generators) is to provide a phase compensation to 180 degrees of the phase of the residues of the inter-area modes to be damped and, next, to increase the gains of the controllers in order to obtain the desired damping for the aforementioned modes. It is shown here that this way of doing is suboptimal. First, from the physical point of view, there is no a priori reason to compensate the phase to 180 degrees. Next, concerning the damping of the oscillations, when the phase compensation is computed in a coordinated way with the gain of the controller, better results are systematically obtained. This means that, in comparison with the standard way of phase compensation (to 180 degrees), this new tuning strategy provide the same damping level obtained with lower gains for the controllers (i.e., with more robust controllers) or, alternatively, higher damping levels with the same level of gains (i.e., better performances). The optimal parameters of the controllers are solutions of constrained optimization problems for the general case of several controllers tuned to damp several modes. Examples on both academic and real large-scale (the European power system model) cases are given. This result is general and can be applied to any control framework (for classic generators or more advanced (optimal) control, for local, inter-area or other oscillatory modes).
\end{abstract}

Keywords: oscillatory modes, PSS/POD tuning, phase lead/lag, optimization.

Preprint submitted to International Journal of Electrical Power Systems ResearchSeptember 21, 2018 


\section{Introduction}

The interconnected power-systems have global oscillatory phenomena which involve several distant devices. When mainly classic synchonous generators are involved, these oscillations are of electromechanical nature (i.e., the most participating parts of the system in the oscillation are the turbine rotors of some generators) and are described by the so-called inter-area modes (see, e.g., [22]). The frequencies of these modes depend on many factors but mainly on the size of the system and the geographic spread of the involved machines. For the European power system, such frequencies actually range between $0.17 \mathrm{~Hz}$ and 0.4 .

The oscillatory modes can be damped using several grid actuators. The most common Damping Controller (DC) are the Power System Stabilizers (PSS) loops of the most participating machines (see, e.g., [24]), but other solutions like the power modulation of the HVDC links [25, 7] or the STATCOMS [17] are also used.

In all cases, the damping principle is to create a swing at the same frequency and in phase opposition with the original oscillation such that the result is a lower damping oscillation (see, e.g., [1], 30]). From the physical point of view, variables in which the oscillation is highly observable and, respectively, controllable are chosen as inputs and, respectively, outputs of the DC. When the latter are used on rotating generators, a brake torque has to be created. For HVDCs, a (active and/or reactive) power modulation is done. From the mathematical point of view, the regulator provides a phase lead or lag and an amplification (see, e.g., 31]). Indeed, the sensitivity of the eigenvalue related to oscillation against the gain of the regulator is proportional with the residue of the open-loop transfer at this eigenvalue. The lead or lag is usually computed to compensate the phase of this residue to 180 degrees and, next, the gain is computed to reach the desired damping. Several techniques exist to compute the phase compensation and the gain - and this can be a complicated task especially when several 
modes should be simultaneous damped using several actuators like, e.g., in [24] - but their great majority are based on the same two-steps principle mentioned above in which the phase is first compensated to 180 degrees.

Advanced robust control has been investigated for this problem [15], 29], [16], 26], 28]. It generally provides better robustness than the classic and direct phase compensation and gain tuning mentioned above 4. For this, a more detailed than the residue information is needed about the system for the control model and this is not an easy task when spread inter-area modes are the target. Indeed, such modes involve a large number of generators which are spread over large zones of the grid which results in high-order control models. Moreover, the resulting controller is usually of dimension equal to the one of the control model and its reduction (by reduction methods for dynamic systems) lower closed-loop performances.

Mixed approaches 21] have been proposed to tune the classic lead-lag structure [31] integrating notions of robustness, like minimization of $H_{\infty}$ norms of key sensitivity and complementary sensitivity transfer matrices [27]. As the structure of the regulator is a priori fixed, robustness items are taken into account as constraints of an optimization problem which need to be solved by numeric iterations (and for which there is no longer analytical solution as for standard $H_{\infty}$ problem) with increased computational burden and difficulty.

In this paper the two-steps (phase lead/lag + gain computation of classic PSS structures 31]) damping principle is revisited both from the optimal and practical points of view. It is shown that the a priori phase compensation at 180 degrees provides suboptimal gains of the regulators. Optimality is here considered from the perspective of the trade-off performances (damping increase)/robustness (small gain in high frequencies, noise attenuation, highorder dynamics tolerance, ...). The goal is thus to improve the classic tuning of fixed standard structures of PSS without going into advanced robust control (like $H_{\infty}$, LMI or the other techniques mentioned above) in order to stay in 
the simple lead-lag formalism and to use only optimization problems easy to solve for enginnering implementation and interpretation facilities. Moreover, from the practical point of view, it is not necessary to ensure a 180 degrees phase compensation since the precise maintain of the frequency of the oscillation is not a priory an issue. Better results are systematically obtained with a coordinated - phase and gain - optimization which integrates minimal notions of robustness and stays simple, i.e., solvable with standard solvers available in toolboxes (like the ones in Matlab) which do not require high engineering knowledge in robust/advanced control. This new method is thus proposed as a good trade-off between performances and robustness on the one hand and complexity (of minimization/synthesis) and accessibility for engineers on the other hand.

The paper is organized as follows: in Section 2 it is briefly recalled the classic two-steps damping principle on a Single Machine connected to Infinite Bus (SMIB) system. Section 3 put into evidence the lack of optimality and presents a new tuning method. This method it is formally developed in Section 4 to cover all the possible situations. A large-scale example of the European power system is also treated in Section 4

\section{Classic damping of oscillatory modes}

\subsection{Inter-area oscillations and PSSs}

Since the development of interconnection of large electric power systems, there have been spontaneous system oscillations at very low frequencies (see, e.g., 32, 20], 19]). They are due to the structure of the system (topology, inertia of the machines, ...). Their frequency is in the electromechanical range and mainly depend on the size of the system and the spread of the mode, i.e., the number and distance among the involved machines. In Europe, the slowest interarea mode is around $0.17 \mathrm{~Hz}$ and involve machines from Turkey which oscillate against machines in the Western part of Europe (Spain, Portugal) [32, [20]. If low damped, they can cause generator outages and even system separation. For 


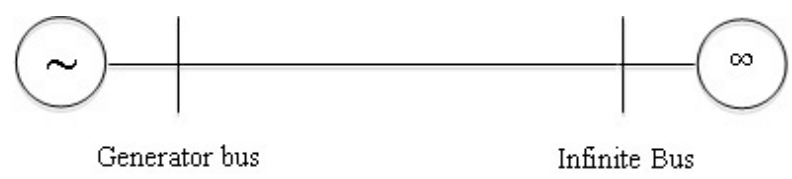

Figure 1: Machine connected to an infinite bus

this reason, damping solutions are in prevention proposed. They are explained using the special modeling presented in the next section.

\subsection{A power system model for the study of inter-area modes}

During low-frequency oscillations, all the electric dynamics of the machine (like the current induced in the damper windings, and the $\mathrm{d}$ and $\mathrm{q}$ natural oscillations) can be neglected, i.e., expressed by algebraic equations in the model. If, moreover, the excitation system is omitted, the so-called classic model of the generator 11] which consists only in the torque differential equation is obtained. For the SMIB system in Fig. 1] the analytic model is in this case

$$
\begin{aligned}
& \frac{d \Delta \delta}{d t}=\Delta \omega_{R} \\
& \frac{d \Delta \omega_{R}}{d t}=\frac{1}{2 H}\left(\Delta P_{m}-\Delta P_{e}-D \Delta \omega_{R}\right) \\
& \Delta P_{e}=K \Delta \delta
\end{aligned}
$$

where $\delta, \omega_{R}, P_{e}, P_{m}, H$ are, respectively, the angle, speed, electrical power, mechanical power and inertia of the machine. $K$ is a constant which depends mainly on the reactance of the line $\left(K=\frac{V_{0}}{X} \cos \delta_{0}\right)$ and $D$ an exogeneous damping factor which mimics the effect of the damping control as explained below.

\subsection{Frequency analysis}

First, one can easily extract from (1) the transfer function

$$
H(s)=\frac{\Delta \delta(s)}{\Delta P_{m}(s)}=\frac{1}{2 H s^{2}+D s+K}
$$

of which poles are

$$
\lambda_{1,2}=\frac{-D \pm j \sqrt{8 H K-D^{2}}}{4 H}
$$




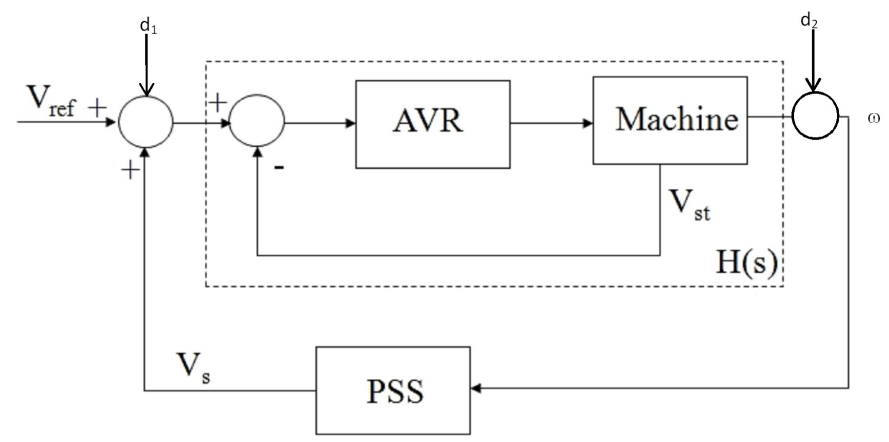

Figure 2: PSS closed-loop

and thus of damping

$$
\xi=D \sqrt{\frac{2 H}{K}} .
$$

(3) gives the only inter-area mode of the system which corresponds to the swing of the generator against the infinite bus (see, e.g., [11] or [22]). The term in (1) which depends on $D$ provides a brake torque if $D>0$. From (4) follows

Fact 1: If a brake torque proportional to $\Delta \omega_{R}$ can be physically produced, this will damp the mode (3).

Fact 1 inspired the control structure in Fig. 2 to damp the mode in which a supplementary PSS loop with input the speed of the machine is added to the already existing AVR.

The frequency of the mode $\sqrt{3}$, i.e., its imaginary part, is $\omega=\sqrt{\frac{K}{2 H}-\frac{D^{2}}{16 H^{2}}}$ and thus $\omega=\sqrt{\frac{K}{2 H}}$ when $D=0$. Notice also that, even when $D \neq 0, \omega \cong \sqrt{\frac{K}{2 H}}$ since $D^{2}$ is neglectable in comparison to $16 H^{2}$. This leads to

Fact 2: Factor $D$ improves the damping of the inter-area mode (3) without changing (a lot) its frequency. 


\subsection{Modal analysis}

The swing mechanism of the inter-area oscillations can be more deeply analyzed using few basic modal tools which are briefly recalled here.

Let

$$
\dot{x}=A x+B u
$$

be a minimal state representation of the open-loop transfer $H(s)$ mentioned above, where $x$ is the state vector of length $n$, i.e.,

$$
H(s)=C(s I-A)^{-1} B .
$$

Equation

$$
\operatorname{det}(\lambda I-A)=0
$$

is referred to as the characteristic equation [22] of matrix A and its solutions are the eigenvalues of matrix A and the poles of $H(s)$. For any eigenvalue $\lambda_{i}$, the $\mathrm{n}$-column vector which satisfies

$$
A \phi_{i}=\lambda_{i} \phi_{i}
$$

is called the right eigenvector of $A$ associated with the eigenvalue $\lambda_{i}$ and, similarly, the $n$-row vector which satisfies

$$
\psi_{i} A=\lambda_{i} \psi_{i}
$$

is called the left eigenvector of $A$ associated with the eigenvalue $\lambda_{i}$. The eigenvectors should be normalized and orthogonal:

$$
\psi_{i} \phi_{j}=1 \text { if } i=j \text { and } \psi_{i} \phi_{j}=0 \text { if } i \neq j .
$$

The participation factors measure the relative participation of the $k$ th state variable in the $i$ th mode:

$$
p_{k i}=\psi_{i k} \phi_{k i}
$$


In view of the eigenvector normalization 110 , the sum of the participation factors associated with any mode $i\left(\sum_{i=1}^{n} p_{k i}\right)$ or with any state variable $x_{i}$ $\left(\sum_{k=1}^{n} p_{k i}\right)$ is equal to 1 . In case of distinct eigenvalues, $H(s)$ can also be expanded in partial fractions as

$$
H(s)=\sum_{k=1}^{n} \frac{r_{k}}{s-\lambda_{k}}+\frac{\bar{r}_{k}}{s-\bar{\lambda}_{k}},
$$

where $r_{k}$ is the residue of $H(s)$ at pole $\lambda_{k}$ and $\bar{\alpha}$ denotes the complex conjugate of $\alpha$.

\section{Remark 1:}

Notice that, in the case of a real power system, $H(s)$ in Fig. 2 must contain not only the dynamics of the machine itself, but also, the relevant dynamics of the overall power system, i.e., the inter-area modes which are concerned. The extraction of this transfer function and the computation of the modal analysis indexes presented above is a difficult task in large-scale and has been studied, for example, in 8 and [13].

Proposition 1 [18, 2]: The sensitivity of a pole $\lambda$ of the closed-loop in Fig. 2 with respect to a parameter $q$ of the transfer function $H_{P S S}(s, q)$ of the regulator PSS is

$$
\frac{\partial \lambda}{\partial q}=\left.r_{\lambda} \frac{\partial H_{P S S}(s, q)}{\partial q}\right|_{s=\lambda}
$$

where $r_{\lambda}$ is the residue of the closed-loop transfer function at pole $\lambda$.

Notice that sensitivity 13 is evaluated for a given value $q_{0}$. The residue $r_{\lambda}$ and the pole $\lambda$ are also computed for this value of the parameter, i.e., for the loop closed with $H_{P S S}\left(s, q_{0}\right)$. If $q_{0}$ is such that $H_{P S S}\left(s, q_{0}\right)=0$ (i.e., for openloop $H(s)$ ), one can directly use Proposition 1 and the open-loop residue as an indicator of the pole shift to achieve the desired damping. More specifically, the situation is as in Fig. 3 where $\lambda=\sigma+j \omega$ is the eigenvalue associated with the inter-area mode which has a damping $\xi$ in open-loop (i.e., Fig. 2 without the 


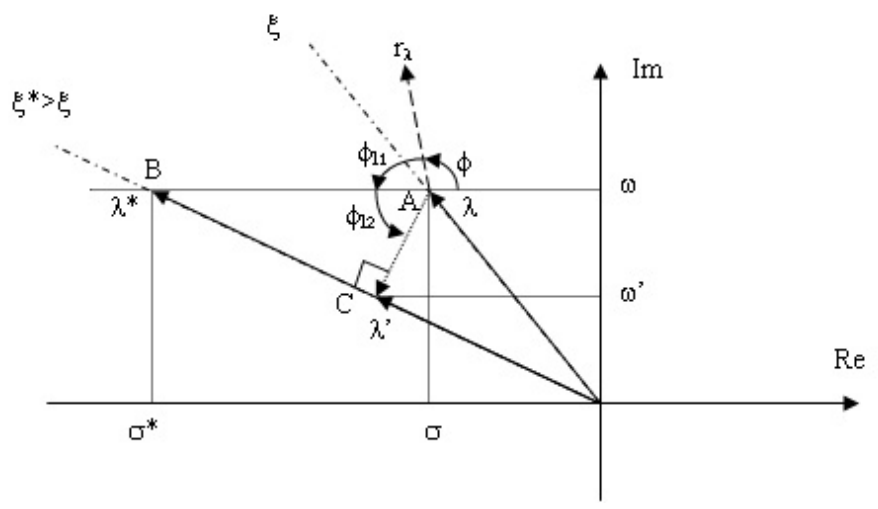

Figure 3: Pole shift

PSS block) and should be shifted to another value $\lambda^{*}$ with desired damping $\xi^{*}$. Only $\xi^{*}$ is specified at the engineering level, so $\lambda^{*}$ has to be found. The usual procedure for this uses Proposition 1 in two steps:

- first, a phase compensation - lead or lag according to the situation- is computed from $\phi$ to 180 degrees, where $\phi$ is the phase of the residu $r_{\lambda}$ of $H(s)$ in $\lambda$. In the case of Fig. 3, this leads to the phase lead $\phi_{l 1}$. To achieve this compensation, $H_{P S S}(s)$ should have a lead/lag block of the form

$$
\left(\frac{1+T_{1} s}{1+T_{2} s}\right)^{p}, p \in \mathbb{N}
$$

- next, a gain $K_{S}$ is computed for the PSS to bring $\lambda$ in the closed-loop to $\xi^{*}$ damping. In the case of Fig. 3 this corresponds to shift from point $A$ to point $B$. Thus

$$
\sigma^{*}=\frac{-\omega \xi^{*}}{\sqrt{1-\xi^{* 2}}}
$$

and 13 along with the phase compensation (14) computed for 180 degrees lead to

$$
K_{S}=\left|r_{\lambda}\right|\left(\sigma^{*}-\sigma\right) .
$$


The resulting structure of the PSS is

$$
H_{P S S}(s)=K_{S}\left(\frac{1+T_{1} s}{1+T_{2} s}\right)^{p}
$$

which is the standard IEEE one 31] which can be enriched by wash-out and torsional filters as shown in 31 .

Fact 3: The PSS structure (17) tuned with the 2-steps procedure above provides the desired damping $\xi^{*}$ for the inter-area mode $\lambda$.

Fact 4: The shift of the eigenvalue from $\lambda$ to $\lambda^{*}$ does not change the mode frequency $\omega$.

\section{Optimality analysis}

The 2-steps method provided above for the tuning of the PSS parameters can be seen as a practical way to introduce a damping factor $D$ into the swing equation of the generator (1) (Facts 1 and 2). Indeed, in both cases, the damping of the mode is increased to a desired (specified) value without changing the frequency of the mode (Facts 2 and 4). Notice that this is a sufficient way of doing but not necessary. Indeed, any shift of the eigenvalue $\lambda$ to the left, i.e., towards the $\xi^{*}$ axis, in the complex plane can be envisaged. From the physical point of view, there is no need to preserve the frequency of the oscillatory mode. The important thing is to achieve the desired damping for this mode. From the mathematical point of view, the horizontal shift does not provide an optimal trade-off between damping improvement and control effort. Indeed, from Section 2, the gain of the regulator is proportional to the length of the shift segment in the complex plane. Under the constraint of constant frequency of the mode (i.e., the shift of $\lambda$ to $\lambda^{*}$ ), this segment is AB in Fig. 3 which is obviously longer than, for example, AC. The shift of the pole to $\lambda^{\prime}$ instead of $\lambda^{*}$ provides the same damping $\xi^{*}$ but at a lower frequency $\omega^{\prime}<\omega$. 
One can thus ask for the optimal eigenvalue shift in the complex plane. For this, the gain and the time constants of the regulator should be simultaneously computed by solving an optimization problem. If the same objectives as before are kept, i.e., to achieve the desired damping $\xi^{*}$ with a minimal control effort, and supplementary robustness requirements are added (see, e.g., [27] for basics of robust control), the idea of the minimum distance criterion given as an example above on Fig. 3 can be used to enhace the framework to the following optimization problem to compute the optimal parameters of the PSS:

$$
\left\{K_{S}{ }^{*}, T_{1}^{*}, T_{2}^{*}\right\}=\operatorname{argmin}\left\{K_{S}\left(\frac{T_{1}}{T_{2}}\right)^{p}\right\},
$$

subject to the following constraints

$$
\begin{aligned}
& \xi \geq \xi^{*} \\
& 0 \leq K_{S} \leq K_{\max } \\
& 0<T_{1}, T_{2} \leq T_{\max } .
\end{aligned}
$$

Notice that $K_{S}\left(\frac{T_{1}}{T_{2}}\right)^{p}$ is the high frequency gain of the PSS 17 and thus related to the open-loop high-gain. The latter should be low in order to improve measurement noise filtering and robustness against neglected dynamics of the power system captured in the transfer model $H(s)$ (see [27]). It is chosen in (18) for the cases where large phase lead $\left(T_{1}>T_{2}\right)$ are needed. This point is treated in more detail in Section 4

\section{Remark 2:}

As in the tuning proposed above the frequency of the mode changes, the settling time will be modified (as it depends on the product of damping and frequency: $T_{s}=-\frac{\delta}{\xi \omega}$, where $\delta$ is the settling purcentage). However, the settling time can be easily included in the specifications if needed as a constraint in 19 (more specifically, modifying the first constraint of 19 which involves only the damping). 


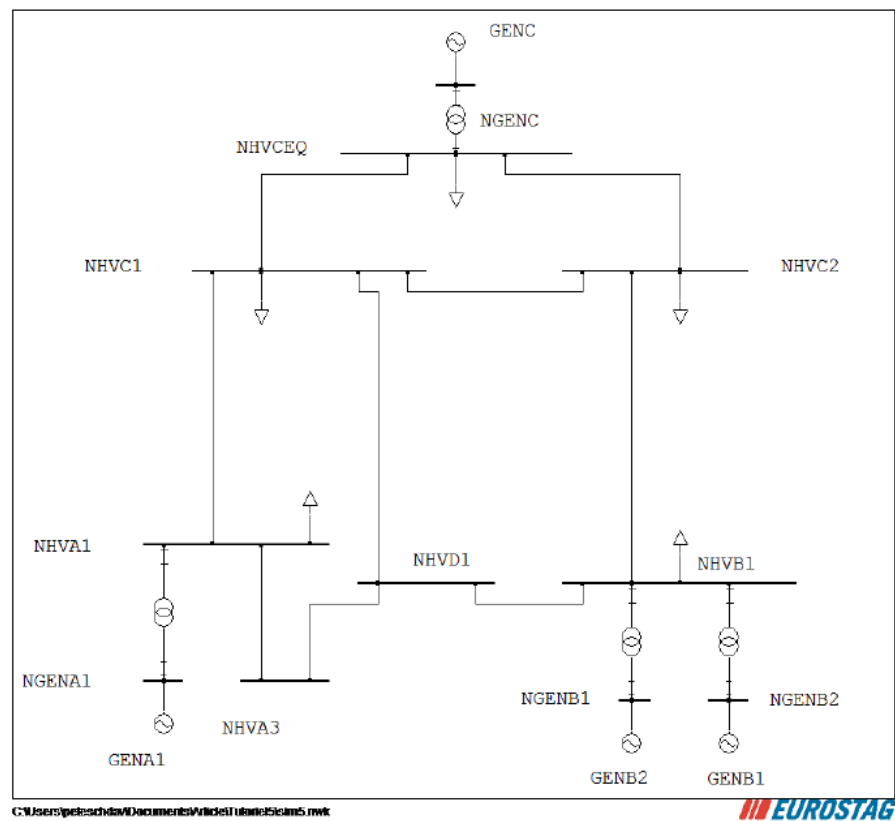

Figure 4: Medium-scale test system

The first constraint in 19 is evaluated by a first-order approximation of the shift of the mode deduced from Proposition 1:

$$
\Delta \lambda=s_{K_{S}} K_{S}+s_{T_{1}} T_{1}+s_{T_{2}} T_{2},
$$

where

$$
\begin{aligned}
& s_{K_{S}}=\frac{\partial H_{P S S}(s)}{\partial K}=r_{\lambda}\left(\frac{1+\lambda T_{1}}{1+\lambda T_{2}}\right)^{p} \\
& s_{T_{1}}=\frac{\partial H_{P S S}(s)}{\partial T_{1}}=p K_{S} r_{\lambda} \lambda \frac{\left(1+\lambda T_{1}\right)^{p-1}}{\left(1+\lambda T_{2}\right)^{p}} \\
& s_{T_{2}}=\frac{\partial H_{P S S}(s)}{\partial T_{2}}=-p K_{S} r_{\lambda} \lambda \frac{\left(1+\lambda T_{1}\right)^{p}}{\left(1+\lambda T_{2}\right)^{p+1}},
\end{aligned}
$$

and $H_{P S S}(s)$ is given by (17). The other constraints in (19) are of usual technological nature.

At this stage, let us apply this new strategy to the power system in Fig. 4 of which parameters are given in Appendix A Full models (nonlinear-7th order, with detailed voltage and frequency regulations) were used for the control and tests for both studied examples (the medium-scale system in this section and 
the large-scale one in the next section). It has an inter-area mode at $0.93 \mathrm{~Hz}$ and with damping $\xi=7.27 \%$ related to the swing of generator GENC against the three machines in the bottom part of the on-line diagram of the system. The modal analysis of this case as well as the large-scale one presented in the next section was done with the SMAS3 package [23]. This mode can be observed in the response in dashed line in Fig. 5 which shows the speed responses of machine GENC to a short-circuit at bus NHVCEQ. The latter simulations as well as the dynamic ones presented in the next section were carried out with the full nonlinear model of the power system in the Eurostag framework [14].

The target damping is $\xi^{*}=10 \%$. The results of (18) with $p=2$ applied to tune the PSS of generator GENA1 are given in the first entries of Table 1 in comparison with the parameters obtained with the classic tuning presented in Section 2 (second entries). It can be seen (for example, in the nonlinear simulations in Fig. 5 that the same damping objective is fulfilled with a lower gain $K_{S}$ of the PSS when the new tuning method is used (solid line). In both cases a lead phase compensation is obtained (column 4 of Table 1) but the gain is lower with the new approach especially in the high frequencies as shown in the Bode plots of the regulators, i.e., of $H_{P S S}$ (17), in Fig. 6 and in the last column of Table 1

Table 1: Comparison of the stabilizer parameters of GENA1 achieved with robust tuning and with standard tuning for the 4-machines case

\begin{tabular}{|c|c|c|c|c|}
\hline$K_{S}{ }^{*}[p u]$ & $T_{1}^{*}[s]$ & $T_{2}^{*}[s]$ & $\phi_{l}[\operatorname{deg}]$ & $K_{H F}[p u]$ \\
\hline $0.0024 / 0.3$ & $10 / 1.58$ & $0.13 / 0.09$ & $108.7 / 118.5$ & $13.2 / 92.4$ \\
\hline
\end{tabular}

The nonlinear simulations reported in Fig. 5 confirm the results of the tuning of the PSS parameters.

Notice also the frequency of the damped mode given in Table 2.

\section{Remark 3:}

Sensitivities 21) are part of the linear model used and are computed around 


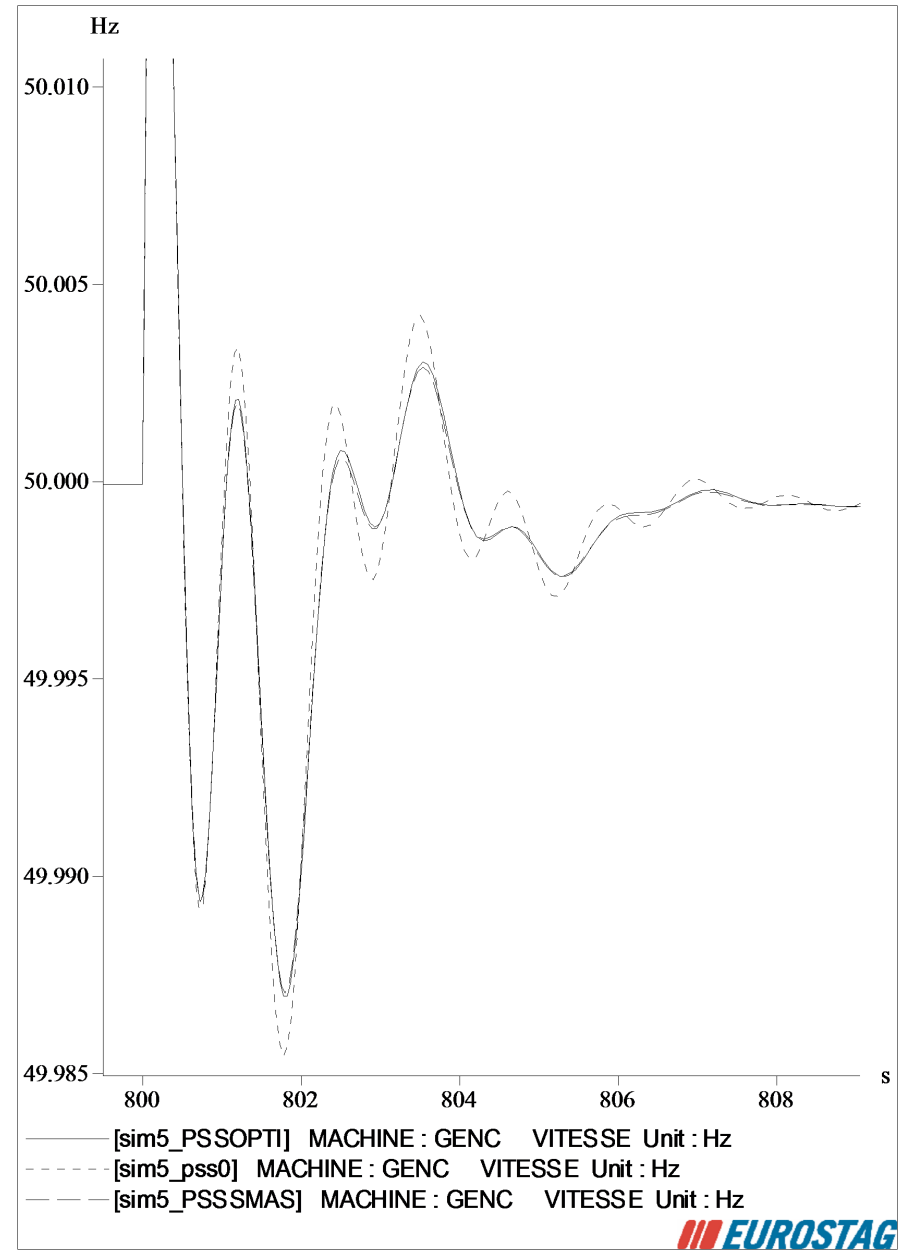

Figure 5: Short-circuit responses of the 4-machines case: solid lines, the optimal tuning ; dash-dotted lines the classic tuning 


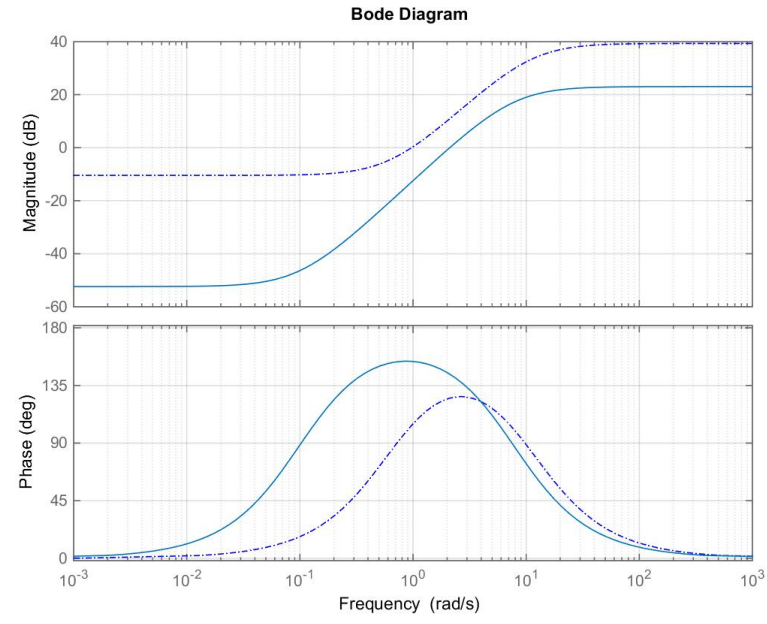

Figure 6: Bode plots of the regulators for the 4-machines case: solid lines, the optimal tuning ; dash-dotted lines the classic tuning

Table 2: Comparison of the frequencies of the mode to be damped

\begin{tabular}{|c|c|c|c|}
\hline & open-loop & classic control & proposed control \\
\hline frequency $[\mathrm{Hz}]$ & 0.93 & 0.93 & 0.94 \\
\hline
\end{tabular}

the equilibrium point obtained in open-loop (i.e., without PSS). Table 3 compares these values with the ones obtained with the loop closed by the obtained PSS regulator. For higher precision, sensitivities might be updated during the iterations but this would also complicate the optimization problem. However, notice that closed-loop sensitivities are much lower (close to zero) that the openloop ones which means that the obtained solution is not far from the optimum and the use of the open-loop sensitivities is an acceptable approximation (tradeoff between simplicity of the optimization and accuracy).

\section{Optimal coordinated tuning for large-scale systems}

The ideas introduced in the previous section are now formalized into an algorithm valid for the general case of large-scale power systems. Several ad- 
Table 3: Comparison of the sensitivities for the 4-machines case

\begin{tabular}{|c|c|c|}
\hline & open-loop & closed-loop \\
\hline $\mathrm{S}_{K_{S}}$ & $-33.7796+\mathrm{j} 3.1204$ & $1.1695+\mathrm{j} 6.4657$ \\
\hline $\mathrm{S}_{T_{1}}$ & $-0.0161+\mathrm{j} 0.0012$ & $-0.0006+\mathrm{j} 0.0031$ \\
\hline $\mathrm{S}_{T_{2}}$ & $0.5036+\mathrm{j} 0.5805$ & $0.1416-\mathrm{j} 0.0512$ \\
\hline
\end{tabular}

justments are thus needed.

First, in the case of a realistic power-system, one spread inter-area mode cannot be damped, in general, with only one PSS. Moreover, in most cases, several inter-area modes have to be damped simultaneously. Thus, the following multivariable form is proposed for equation 20

$$
\Delta \lambda_{j}=\sum_{i=1}^{m}\left(s_{K_{S_{i}}} K_{S_{i}}+s_{T_{1}^{i}} T_{1}^{i}+s_{T_{2}^{i}} T_{2}^{i}\right), j=1, \ldots, l,
$$

where $l$ is the number of modes to be damped and $m$ the number of PSSs to be tuned for this and the same kind of sensitivities (13) and constraints (19) are used.

This situation is illustrated on a realistic large-scale representation of the interconnected European power system. More precisely, this is a representation of the European power system before the interconnection with zone 2 (Romania and Bulgaria) and Turkey. It consists of about 2000 buses, 2400 lines and 810 transformers. The generators with power greater than 100MW (about 400 machines) are represented by detailed dynamic models along with the detailed models of their regulations. The rest of the generation is considered as static injection at the load-flow stage. A winter peak load scenario is considered. The resulting linear model is described by about 8000 state variables. It is wellknown that this system exhibits a low damped inter-area oscillation around 0.22 $\mathrm{Hz}$ in which the generators of the eastern part of the grid are oscillating against the generators of the western part [3]. This phenomenon is represented by the 
first two modes of the linearized full model of which dampings are given in the first line of Table 4 They are studied in this paper along with the one in the third column of the same table which is of different nature; it is an inter-area mode of the Spanish system at a slightly higher frequency $(0.9 \mathrm{~Hz})$ than the first two ones. It is chosen here along with the slow ones $(\sharp 1$ and $\sharp 2)$ in order to put into evidence the coordination needed in the tuning for damping inter-area modes in different frequency ranges. Thus, the target is to simultaneously ensure a 10\% damping for the three modes in Table 4 . This objective is interpreted as follows: mode \#1 is poorly damped and $\xi_{1}$ should thus be increased, but the damping actions should be chosen in order to not degrade the damping of the other two modes, one directly concerned by the East-West oscillation and the other one local to the Spanish system.

Table 4: Damping $\xi[\%]$ of the East-West modes of the European system

\begin{tabular}{|c|c|c|c|}
\hline & mode $\sharp 10.23 \mathrm{~Hz}$ & mode $\sharp 20.24 \mathrm{~Hz}$ & mode $\sharp 30.91 \mathrm{~Hz}$ \\
\hline without PSSs & 3.8 & 11.7 & 6.2 \\
\hline conv. PSSs & 12 & 9.1 & 7.8 \\
\hline optim. PSSs & 12 & 9.1 & 7.8 \\
\hline
\end{tabular}

Next, the objective function in 18 should be enriched to cover all the situations. Indeed, in Section 3 only the situation when a phase lead is needed for the PSS was considered. As a matter of fact, the optimal computation of $T_{1}$ and $T_{2}$ can lead to a lag of phase if $T_{1}<T_{2}$. Notice that, in the classic tuning method recalled in Section 2, this is encountered when the residue of the eigenvalue to be shift is of negative phase. In this case, the high frequency gain of the PSS is not an issue for robustness which led us to the following optimisation problem in the general case

$$
\left\{K_{S_{i}}^{*}, T_{1_{i}}^{*}, T_{2_{i}}^{*}\right\}_{i=1, \ldots, m}=\operatorname{argmin}\left\{J\left(K_{S_{i}}, T_{1_{i}}, T_{2_{i}}\right)\right\}
$$


where

$$
J\left(K_{S_{i}}, T_{1_{i}}, T_{2_{i}}\right)=\sum_{j} K_{S_{j}}+\sum_{k} K_{S_{k}}\left(\frac{T_{1_{k}}}{T_{2_{k}}}\right)^{p}
$$

with $j \in\{1, \ldots, m\}$ such that $T_{1_{j}} \leq T_{2_{j}}$ and $k \in\{1, \ldots, m\}$ such that $T_{1_{k}}>$ $T_{2_{k}}$.

Notice that (24) is the transposition of the robustness loop-shaping concepts to our particular fixed structure PSS tuning problem. In case of need for phase lead, $T_{1}$ and $T_{2}$ should respect high-frequency gain specification. In case of phase lag, they are free which leads to phase margin improvement.

For the European test system presented above, the Spanish machines Almaraz, Cofrentes and PGR were chosen to damp the modes selected in Table 1 since they have high participation in these modes and they are not already equipped with PSSs.

Table 5: Comparison of the stabilizer parameters achieved with robust coordinated tuning and with classic coordinated tuning for damping of 3 modes using 3 machines of the European system

\begin{tabular}{|c|c|c|c|c|}
\hline & $K_{S}{ }^{*}[p u]$ & $T_{1}^{*}[s]$ & $T_{2}^{*}[s]$ & $K_{H F}[p u]$ \\
\hline Almaraz & $0.12 / 2.43$ & $10 / 0.21$ & $1.43 / 0.02$ & $6.17 / 243$ \\
\hline Cofrentes & $0.0055 / 5.74$ & $10 / 0.58$ & $0.1 / 0.05$ & $47.88 / 574$ \\
\hline PGR & $0 / 0.54$ & $/ 0.6$ & $/ 0.06$ & $0 / 54$ \\
\hline
\end{tabular}

The dampings achieved with the two-phases multivariable coordinated tuning method 24] based on a 180 degrees phase compensation (the latter coordinated tuning method is briefly recalled in Appendix B for the three inter-area modes are given in row 3 of Table 4 . Notice that the target damping $\xi^{*}=10 \%$ it is not achieved for all 3 modes. This is mainly due to the fact that the number of PSSs chosen for the tuning it is not sufficient to achieve such a level of damping. In practice, the desired level could be achieved if more PSSs are 
used. Here, as we are interested only in comparing the performance level of the two tuning methods, we adopt as target damping of the new proposed optimal tuning method the results of the classic tuning mentioned above, i.e., the values in the row 3 of Table 4 First, these performance objectives are feasible (as satisfied with the classic controller). Next, if achieved with the new control, one could directly compare robustness level and implementation facts for the two controllers (which ensure the same level of performance). The parameters tuned with (23) for this target are given in the first entries of Table 5 in comparison with the ones in the second entries obtained with [24]. Indeed, the damping target is fulfilled (row 4 of Table 4). Also, only 2 PSS (instead of 3 with the calssic tuning) are needed to provide the same damping level (the gain $K_{S}$ obtained for PGR is zero).

Next, as lead phase is obtained for all the controllers, a measure of the robustness is the high frequency gain for the tuned PSSs given in the last column in Table 5 or their total (sum) which is 54.05 with the new proposed tuning method and 871 with the classic one, thus sensibly lower with the new method. The global frequency profile of the three PSSs are given in Figs. 7, 8 for both controls (solid lines for the new controllers, dash-dotted ones for the classical ones).

Figs. 9 and 10 show the nonlinear time-responses to a short-circuit which excites one of the east-west inter-area modes. They confirm the results of the linear analysis above. Notice also that, even if the linear estimated a posteriori damping level is the same with the two tuning methods (this was the scope in order to make possible the comparison of the performances and robustness), the effective damping achieved in full nonlinear simulation in Figs. 9 and 10 with the new method is higher with the new method.

\section{Remark 4:}

The optimization problem (23) has been solved with standard Matlab routines of the Optimization toolbox at this stage. For further applications to more 


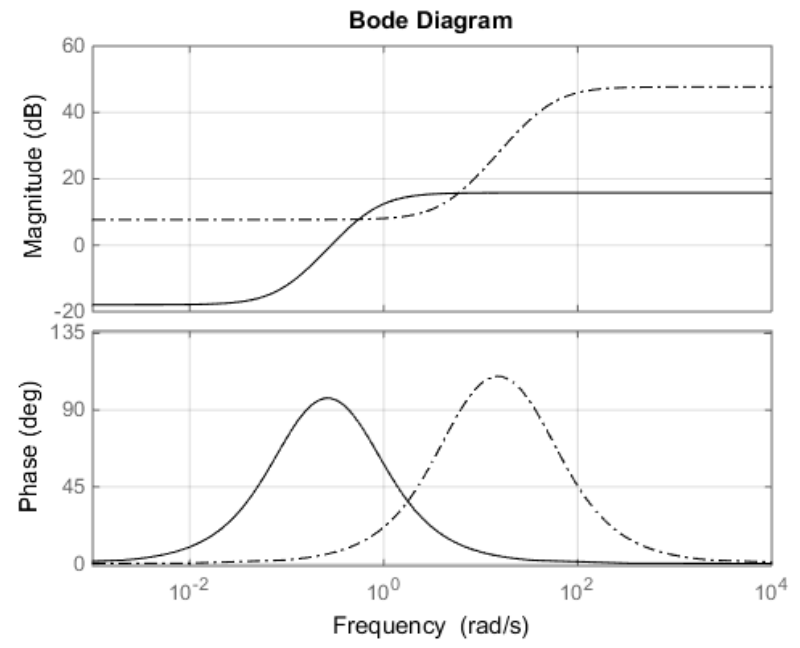

Figure 7: Almaraz Bode plots of the regulators for the European case: solid lines, the optimal tuning ; dash-dotted lines the classic tuning

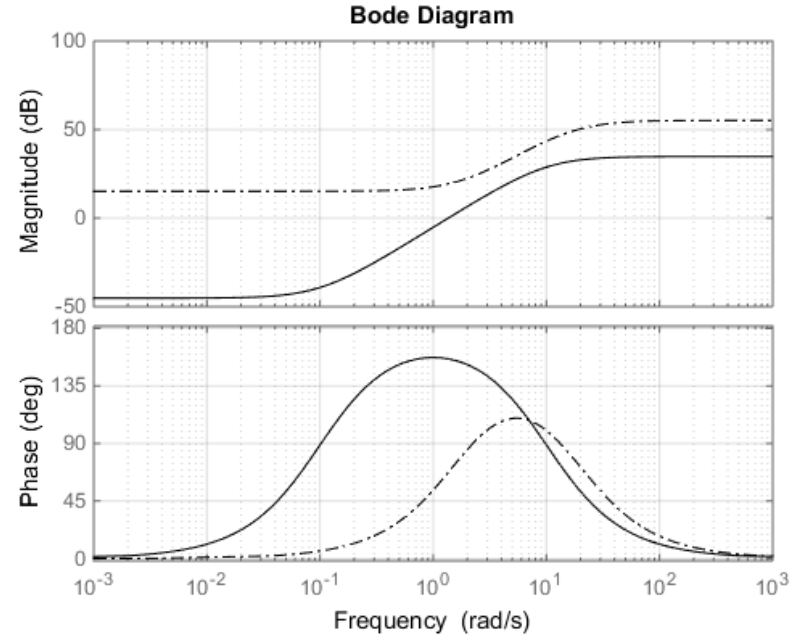

Figure 8: Cofrentes Bode plots of the European case 


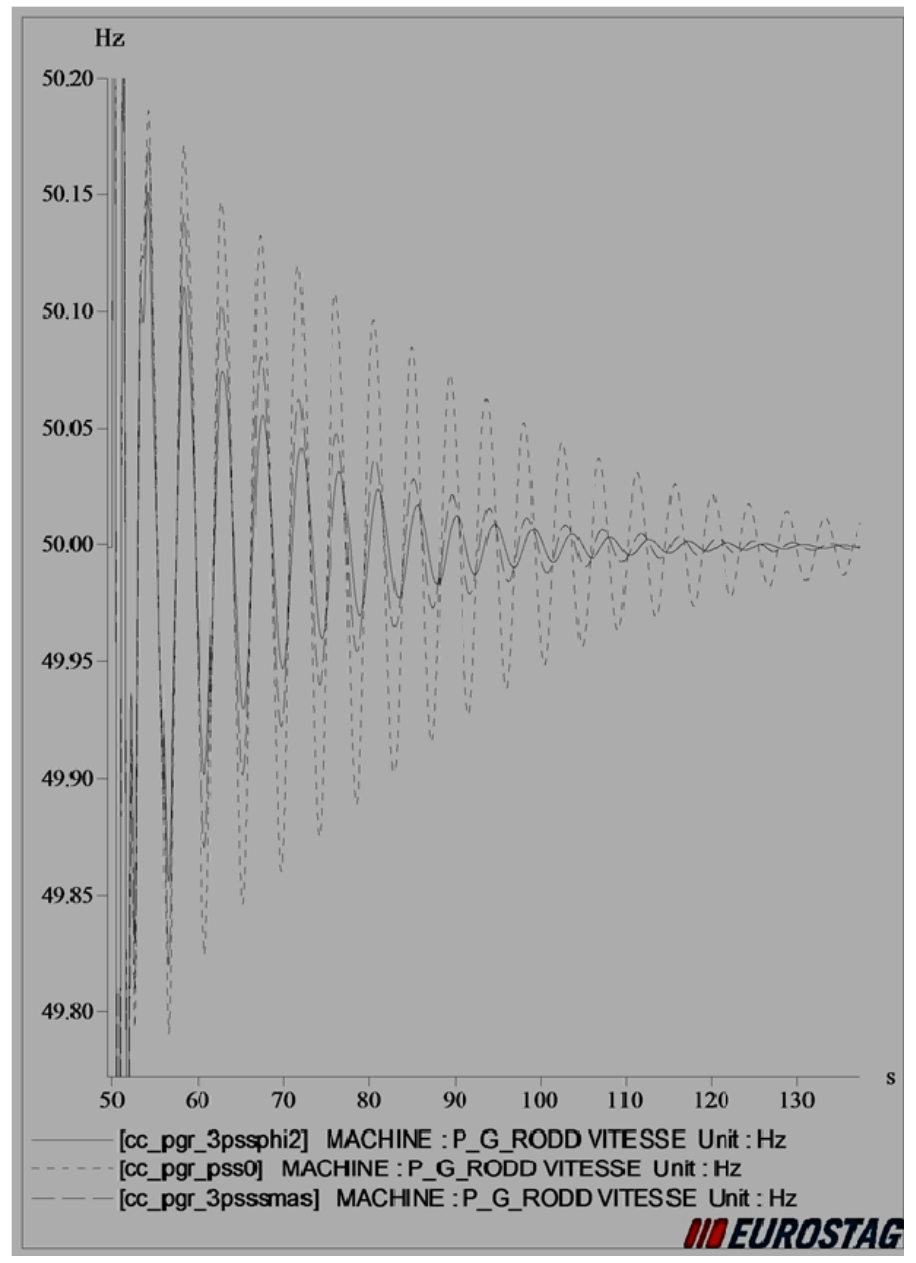

Figure 9: Short-circuit responses for coordinated PSS Cofrentes 


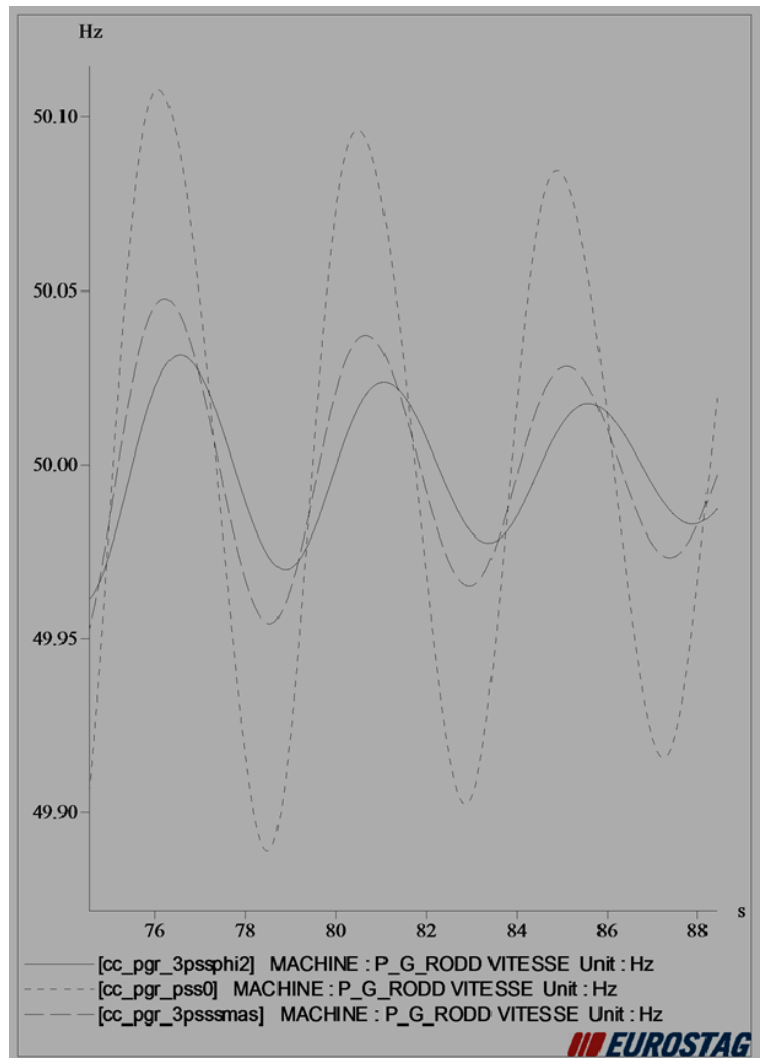

Figure 10: Zoom of short-circuit responses Bode plots for coordinated PSS Cofrentes 
complex cases, algorithms with better performances may be used. Indeed, the optimization methodologies have been the subject of ongoing enhancements over the years (see, e.g., [6]), [5]).

\section{Robustness}

\subsection{Unstructured robustness and disturbance rejection}

Consider disturbances $d_{1}$ and $d_{2}$ on the closed-loop in Fig. 2. From a technological point of view, the input disturbance $d_{1}$ corresponds to inherent actuator faults (offset, failure, parasitic signal, ...). The output disturbance $d_{2}$ can be due to meter disfunction (failure or bias) and/or measurement noise. From a system point of view, these signals may capture exogeneous dynamics not taken into account in the nominal control model. This is classic for robust control [27. For the case of the interconnected power systems, $d_{1}$ may account for voltage/electric dynamics whereas $d_{2}$ stands for all speed/frequency disturbance (coming from load/production imbalance, oscillatory phenomena of other nature than inter-area modes, like, e.g., subsyncronous oscillations, higher order harmonics, ...). As the control model is low frequency (focused on inter-area modes), such neglected dynamics are of higher frequency. Here, they are considered at $10 \mathrm{rad} / \mathrm{sec}$, generated as a step response of a second-order element tuned to this frequency. In other words,

$$
d_{i}=\frac{\omega^{2}}{s^{2}+2 \xi \omega s+\omega^{2}} u_{i}, i \in\{1,2\},
$$

where $\omega=10 \mathrm{rad} / \mathrm{sec}, \xi=0.0025, u_{1}$ is a step of magnitude $0.1 \mathrm{pu}$ and $u_{2}$ a step of magnitude $0.01 \mathrm{pu}$.

Fig. 11 shows speed responses of the generator equipped with the PSS with classic (dotted line) and proposed regulator (solid lines) closed-loops of the first example (Fig. 4) to such an input disturbance. The effect of $d_{2}$ is shown in Fig. 12. In both cases, the loop closed with the proposed regulators better rejects the disturbances. This difference is even more pregnant for higher frequencies (like 

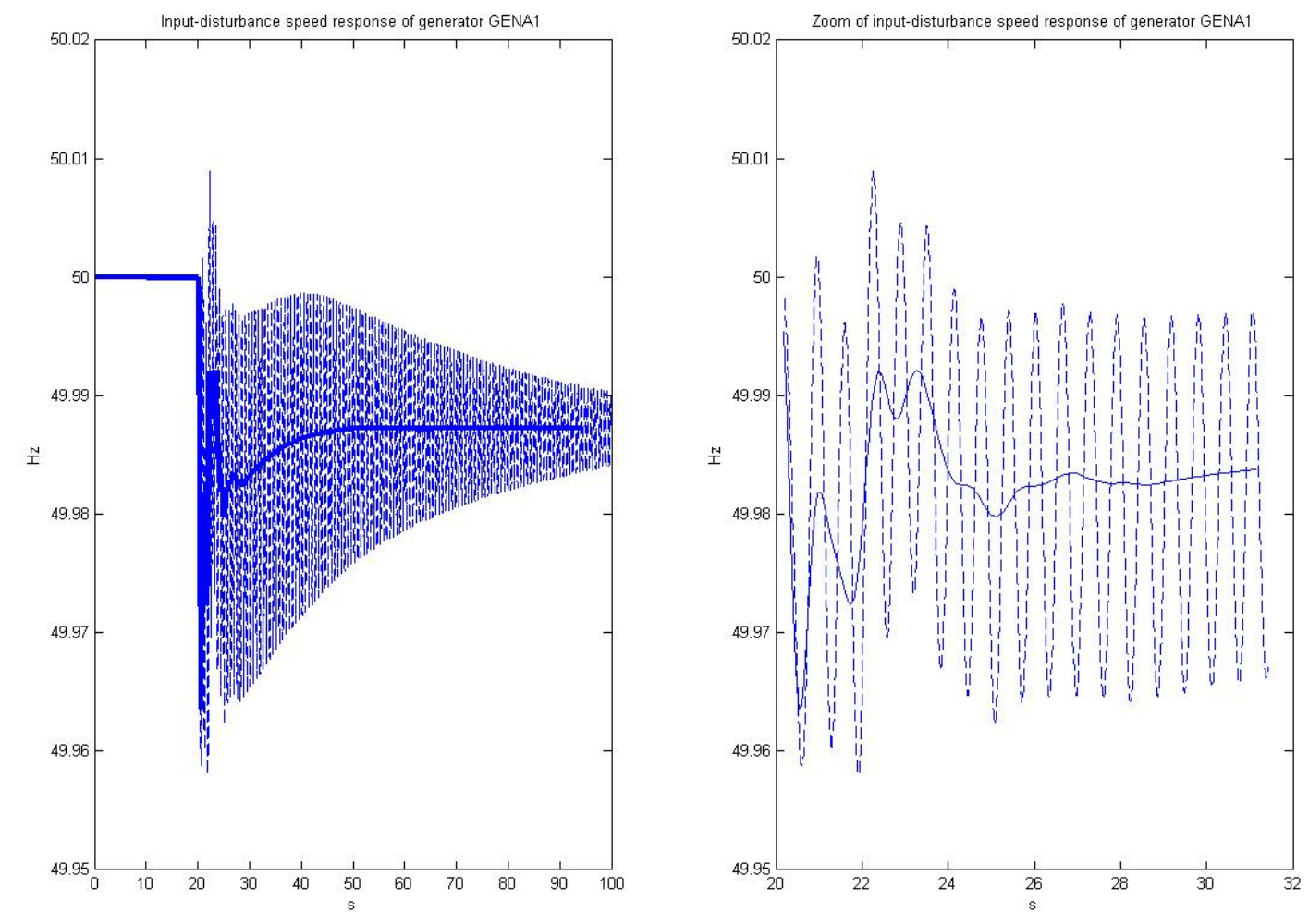

Figure 11: Input disturbance rejection

for noises). The test is run here with such quite low frequency to account not only for noises and high-order harmonics but also for other electric (unmodeled) dynamics as mentioned before.

\subsection{Structured robustness}

Such robustness concerns variations of the parameters of the control model. In our case, these may be due to load/generation evolution or tripping of grid elements. Fig. 13 shows the responses of the same first example in 2 cases of line tripping: line NHVC1-NHVA1 for the left subfigure and line NHVC1-NHVD1 for the right subfigure. Both lines are in the direct path of the oscillation of the 

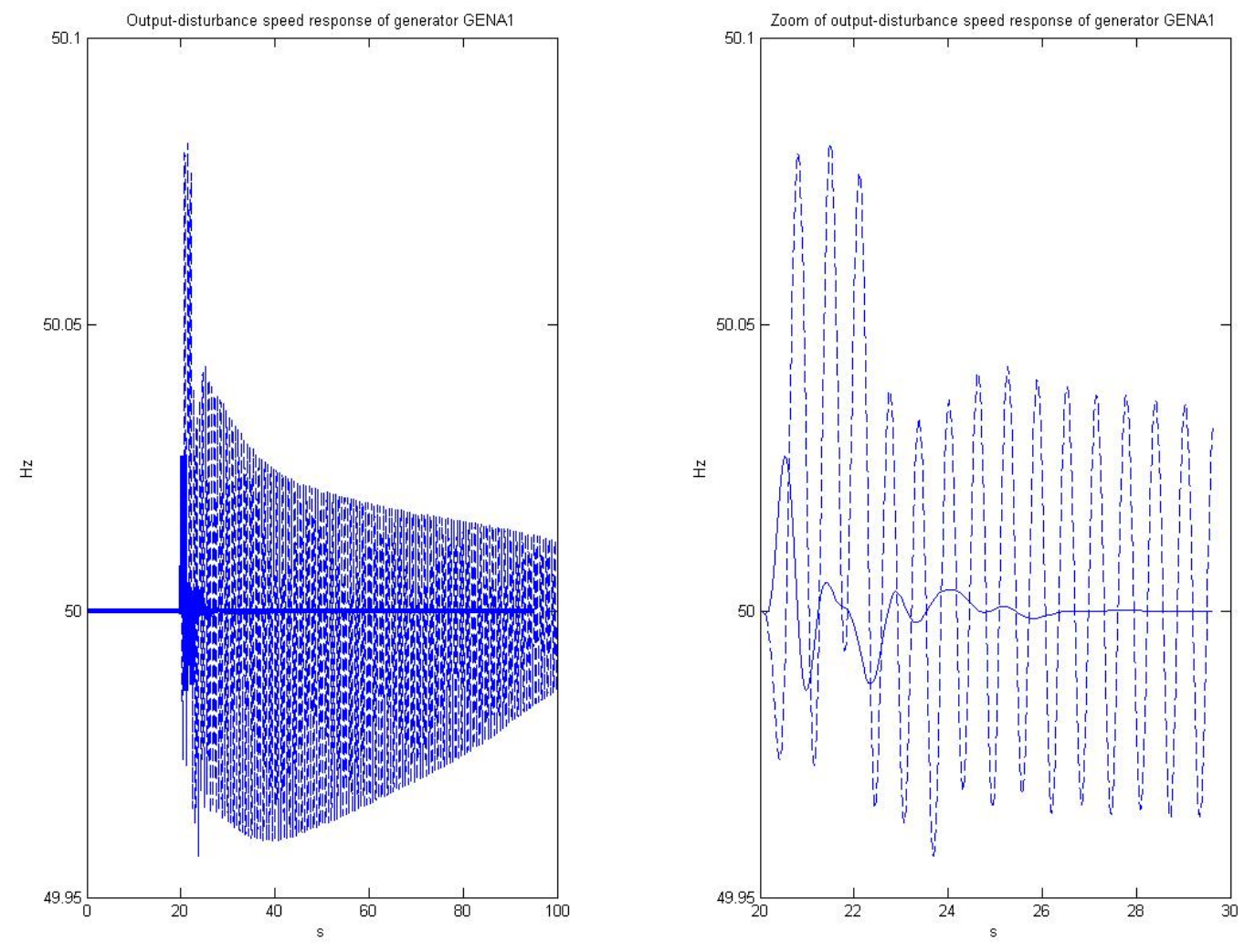

Figure 12: Output disturbance rejection 

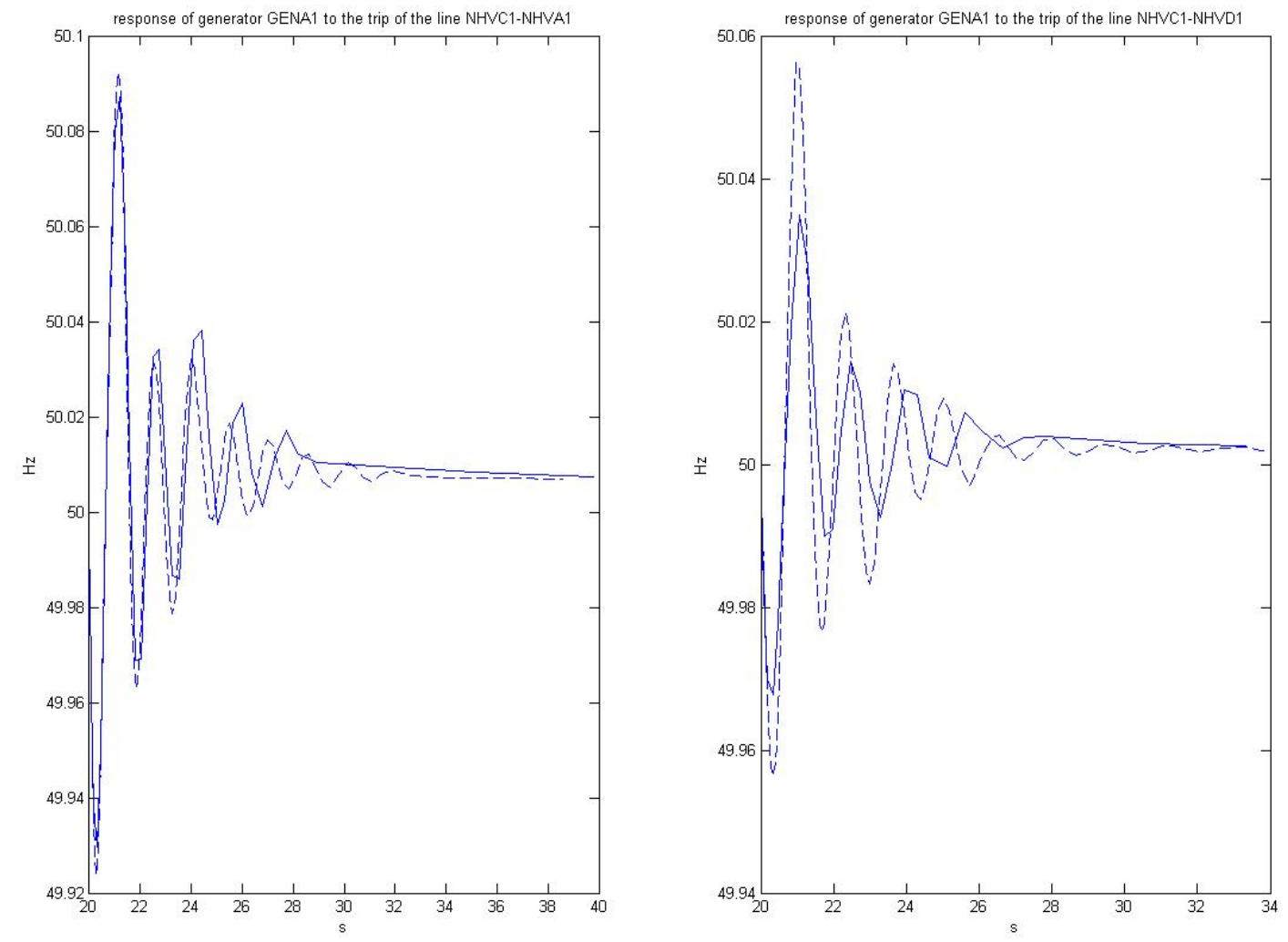

Figure 13: Line trip

inter-area mode to be damped. It is well known (see, e.g., [5]) that topological modification of such corridor alter the inter-area mode and it is thus a good robustness test. In both situations, better responses are obtained with the proposed controller in comparison to the classic one (Fig. 13).

\section{Conclusion}

It has been shown that the 180 degrees phase compensation usually used in the tuning of the parameters of power oscillations damping controller is sub- 
optimal. If the phase compensation is computed in conjunction with the gains via a coordinated optimization problem, better results are obtained. For the case of several controllers tuned to damp several modes, even with the phase compensation constrained to 180 degrees, an optimization is needed for the computation of the gains in a coordinated manner (see, e.g., [24]). The methodology proposed here needs thus no extra effort for the implementation. It was tested for the tuning of PSS loops of classic synchronous generators but it is valid for the tuning of any structure of regulator for any damping actuator (for example, the power modulation of HVDC links or other FACTS). A new optimization criterion which integrates some basic robustness principles has been proposed to illustrate the advantages against the constraint on the phase compensation to 180 degrees. This approach can be applied for any oscillatory modes: inter-area, local, intraplant or electrical coupling ones [1]. The robustness demand can be further improved by several means like in, e.g., [9], [10, [12.

\section{Appendix A. Data of the medium-scale test system (Fig. 4)}

$$
\text { generators' parameters (no load pu): }
$$

stator resistance $0.005 \mathrm{pu}$, stator leakage $0.219 \mathrm{pu}$, direct reactance $2.57 \mathrm{pu}$, direct transient reactance $0.422 \mathrm{pu}$, direct sub-transient reactance $0.3 \mathrm{pu}$, direct transient time constant $7.695 \mathrm{~s}$, direct sub-transient time constant $0.061 \mathrm{~s}$, quadrature reactance $2.57 \mathrm{pu}$, quadrature transient reactance $0.662 \mathrm{pu}$, quadrature subtransient reactance $0.301 \mathrm{pu}$, quadrature transient time constant $0.643 \mathrm{~s}$, quadrature sub-transient time constant $0.095 \mathrm{~s}$, inertia 4MWs/MVA

step-up transformers: $24 \mathrm{kV} / 380 \mathrm{kV}$, rate $=1.1 \mathrm{pu}, \mathrm{X}=0.00769 \mathrm{pu}$ for GENA1, $\mathrm{X}=0.02251 \mathrm{pu}$ for GENB1, $\mathrm{X}=0.09 \mathrm{pu}$ for GENB2, $\mathrm{X}=0.00769 \mathrm{pu}$ for GENC (base $24 \mathrm{kV}$ )

regulators:

AVR of each machine: $E F D=K_{V} \frac{K_{e}}{1+s T_{e}}\left(V_{r e f}-V_{t}\right)$ with $K_{e}=1 \mathrm{pu} ; \mathrm{K}_{V}=$ 
$40 p u ; T_{e}=0.1 \mathrm{~s}$ for GENA1 and GENB1 and $\mathrm{K}=30 \mathrm{pu} ; T_{e}=1 \mathrm{~s}$ for GENB2 and GENC

GOVERNOR for all the machines: $T_{m}=\frac{1}{1+0.1 s} \frac{1}{1+0.5 s} \frac{1+3 s}{1+10 s}(1-\omega)$

standard PSS of machine GENC: $K_{S}=1.07 p u, T_{1}=3.11 s, T_{2}=0.73 \mathrm{~s}$

optimal PSS of machine GENC: $K_{S}=4.32 p u, T_{1}=3.09 \mathrm{~s}, T_{2}=1.67 \mathrm{~s}$

generation and load:

NGENA1: $\mathrm{P}=900 \mathrm{MW}, \mathrm{Q}=300 \mathrm{MVAR}, \mathrm{V}=24 \mathrm{kV}$

NGENB1: $\mathrm{P}=400 \mathrm{MW}, \mathrm{Q}=190 \mathrm{MVAR}, \mathrm{V}=24 \mathrm{kV}$

NGENB2: $\mathrm{P}=900 \mathrm{MW}, \mathrm{Q}=300 \mathrm{MVAR}, \mathrm{V}=24 \mathrm{kV}$

NGENC: $\mathrm{P}=2504 \mathrm{MW}, \mathrm{Q}=1500 \mathrm{MVAR}, \mathrm{V}=24 \mathrm{kV}$

NHVA1: $\mathrm{P}=-1000 \mathrm{MW}, \mathrm{Q}=-100 \mathrm{MVAR}$

NHVB1: $\mathrm{P}=-1000 \mathrm{MW}, \mathrm{Q}=-300 \mathrm{MVAR}$

NHVC1: $\mathrm{P}=-500 \mathrm{MW}, \mathrm{Q}=-100 \mathrm{MVAR}$

NHVC2: $\mathrm{P}=-600 \mathrm{MW}, \mathrm{Q}=-200 \mathrm{MVAR}$

NHVCEQ:P=-700MW, Q=-150MVAR

line reactances $[\mathrm{pu}]\left(S_{\text {base }}=100 \mathrm{MVA}\right.$ and $\left.V_{\text {base }}=380 \mathrm{kV}\right)$ :

NHVC1-NHVA1: $0.490 \quad$ NHVC2-NHVB1: 0.5

NHVD1-NHVC1: 0.500 NHVC1-NHVC2: 0.011

NHVD1-NHVB1: 0.011 NHVCEQ-NHVC1 and 2: 0.22

NHVA3-NHVD1: 0.011 NHVA3-NHVA1: 0.02 


\section{Appendix B. Coordinated tuning method with phase compensation to 180 degrees $[24$}

The design is carried out in two consecutive steps:

- first, the phase compensation of each PSS is computed such that the phase of the eigenvalues sensitivities becomes as closer as possible to 180 degrees. If

a filtering ratio $\alpha_{i}=\frac{T_{1_{i}}}{T_{2_{i}}}$ is assumed for each PSS $i$, the following problem is solved

$$
\begin{aligned}
& \left\{T_{1_{i}}^{*}\right\}_{i=1, \ldots, m}=\operatorname{argmin}\left\{\sum_{j=1}^{p} \beta_{i j} \cos \left[\arg \left(S_{j}\left(T_{1_{i}}\right)\right]\right\}\right. \\
& T_{2_{i}}^{*}=\frac{T_{1_{i}}^{*}}{\alpha_{i}},
\end{aligned}
$$

where $\beta_{i j}=\frac{\left|r_{i j}\right|}{\sum_{k=1}^{p}\left|r_{k j}\right|}, S_{j}\left(T_{1_{i}}\right)=\left.\frac{\partial H_{P S S_{i}}(s)}{\partial K_{S_{i}}}\right|_{s=\lambda_{j}}$ and $H_{P S S_{i}}(s)$ is of form (17) for each PSS $i$.

- next, the gains of the controllers are determined to shift the eigenvalues up to the desired damping. The gains of the controllers are determined by solving another programming problem of which objective function is to minimize the control action. The latter is expressed as the sum of the gains weighted by the sensitivities:

$$
\left\{K_{S_{i}}^{*}\right\}=\operatorname{argmin}\left\{\sum_{j=1}^{m} \gamma_{j} K_{S_{j}}\right\},
$$

where $\gamma_{j}=\sum_{k=1}^{n}\left|\frac{\partial \lambda_{k}}{\partial K_{S_{j}}}\right|$.

\section{Acknowledgment}

The author would like to thank Pr. L. Rouco, IIT-Universidad Pontificia Madrid for discussions on the manuscript.

[1] L. Arioua, B. Marinescu, Robust grid-oriented control of high voltage DC links embedded in an AC transmission system, Int. J. Robust Nonlin. Contr., 26, 1944-1961, 2016. 
[2] I-P. Arriaga, J-C. Verghese, L. Pagola and F.C. Schweppe, "Developments in Selective Modal Analysis of Small-signal Stability in Electric Power Systems", Automatica, Vol. 26, No. 2, pp. 215-231, 1990.

[3] H. Breulmann et al., "Analysis and Damping of Inter-area Oscillations in the UCTE-CENTREL Power-System", CIGRE Session, Paper 38-113, Paris, 2000.

[4] G.E. Boukarim, S. Wang, J.H. Chow, G.N. Taranto and Nelson Martins, "A Comparison of Classical, Robust, and Decentralized Control Designs for Multiple Power System Stabilizers", IEEE Trans. on Power Systems, Vol. 15, No. 4, pp. 1287-1292, November 2000.

[5] A.L.B. do Bomfim, G.N. Taranto and D.M. Falcao, "Simultaneous Tuning of Power System Damping Controllers Using Genetic Algorithms", IEEE Trans. on Power Systems, Vol. 15, No. 1, pp. 163-169, February 2000.

[6] M.R. Shakarami, I. Faraji Davoudkhani, "Wide-area power system stabilizer design based on Grey Wolf Optimization algorithm considering the time delay", Electric Power Systems Research, Volume 133, pp. 149-159, April 2016.

[7] C. Guo, W. Liu, C. Zhao and X. Ni, "Small-signal dynamics and control parameters optimization of hybrid multi-infeed HVDC system", Int. J. of Electrical Power and Energy Systems, vol. 98, pp. 409-418, 2018.

[8] I. Kamwa, G. Trudel, L. Gerin-Lajoie, "Low-Order Black-Box Models for Control System Design in Large Power Systems", IEEE Trans. on Power Systems, Vol. 11, No. 1, pp. 303-311, February 1996.

[9] I. Kamwa, G. Trudel and D. Lefebvre, "Optimization-Based Tuning and Coordination of Flexible Damping Controllers for Bulk Power Systems", Proc. of IEEE International Conference on Control Applications, August 1999. 
[10] I. Kamwa, G. Trudel and L. Gérin-Lajoie, "Robust Design and Coordination of Multiple Damping Controllers Using Nonlinear Constraints Optimization", IEEE Trans. on Power Systems, Vol. 15, No. 3, August 2000.

[11] P. Kundur, Power System Stability and Control, McGraw-Hill, 1994.

[12] B. Marinescu, B. Mallem, H. Bourlès and L. Rouco, "Robust Coordinated tuning of Parameteres of Standard Power Systems Stabilizers for Local and Global Grid Objectives", Proc. of Power Tech Conference, June 28th-July 2nd, Bucharest, Romania, 2009.

[13] B. Marinescu and D. Petesch, "Three-level coordination in power system stabilization", Int. J. of Electric Power Systems Research, Vol. 111, pp. 40$51,2014$.

[14] B. Meyer and M. Stubbe, "Eurostag, a Single Tool for Power System Simulation", Proc. of IEEE Conf. on Transmission and Distribution, March 1992.

[15] D. Mondal, A. Sengupta, A. Chakrabarti, "Robust Control of Inter-area Oscillations in a Multimachine Network Employing LMI Based Wide Area TCSC Controller", Electrical and Electronic Engineering, 2(2): pp. 23-30 , 2012.

[16] K. Mekki, N. Hadjsaid, R. Feuillet and D. Georges, "Design of Damping Controllers of Inter-Area Oscillations in a Multimachine Network Employing LMI Based Wide Area TCSC Controller", Proc. of IEEE PES Int. Conf. on Power Ind. Computer Applications (PICA), 2001.

[17] M.H. Haque, "Damping improvement by FACTS devices: A comparison between STATCOM and SSSC", Electric Power Systems Research, Volume 76, Issues 910, pp. 865-872, June 2006.

[18] F.L. Pagola, I.J. Pérez-Arriaga and G.C. Verghese, "On Sensitivities, Residues and Participations: Applications to Oscillatory Stability Analy- 
sis and Control", IEEE Trans. on Power Systems, Vol. 4, No. 1, February 1989.

[19] A. Pal, J.S. Thorp, S.S. Veda and V.A. Centeno, "Applying a robust control technique to damp low frequency oscillations in the WECC", Int. J. of Electrical Power and Energy Systems, Vol. 44, Issue 1, pp. 638-645, 2013.

[20] I.A. Nassar and H. Weber, "System analysis of the Turkish power system for interconnection with continental Europe Power Plants and Power Systems Control",Proc. of The 8th Power Plant and Power System Control Symposium, Toulouse-France, 2012.

[21] D. Rimorov, I. Kamwa and Geza Joós, "Quasi-Steady-State Approach for Analysis of Frequency Oscillations and Damping Controller Design", IEEE Trans. on Power Systems, Vol. 31, No. 4, July 2016.

[22] G. Rogers, Power Systems Oscillations, Kluwer, 2000.

[23] L. Rouco, I.J. Pérez-Arriaga, R. Criado and J. Soto, "A computer Package for Analysis of Small Signal Stability in Large Electric Power Systems", Proc. of the 11th Power Systems Computations Conference, pp. 1141-1148, Avignon (France), August 1993.

[24] L. Rouco, "Coordinated Design of Multiple Controllers for Damping Power System Oscillations", International Journal of Electrical Power and Energy Systems, Vol.23, No.7, pp. 517-530, October 2001.

[25] S-Y. Ruan, G-J. Li, T.T. Lie and S-S. Choi, "Improving power system damping by utilizing VSC-HVDC", Proc. of IEEE PES General Meeting, 2012.

[26] J-K. Shiau, G.N. Taranto, J.H. Chow and George Boukarim, "Power Swing Damping Controller Design Using an Iterative Linear Matrix Inequality Algorithm", IEEE Trans. on Control Systems Technology, Vol. 7, No. 3, pp. 371-381, February 1999. 
[27] S. Skogestad and I. Postlethwaite, Multivariable Feedback Control, John Wiley and Sons Ltd., Chichester-England, 1996.

[28] G.N. Taranto and J.H. Chow, "A Robust Frequency Domain Optimization Technique for Tuning Series Compensation Damping Controllers", IEEE Trans. on Power Systems, Vol. 10, No.3, August 1995.

[29] K. Vance, A. Pal and J.S. Thorp, "A Robust Control Technique for Damping Interarea Oscillations", Proc. of Power and Energy Conference at Illinois (PECI), 2012.

[30] Y-N. Yu, Electric Power System Dynamics, Academic Press, 1983.

[31] IEEE Power Engineering Society, "IEEE Recommended Practice for Excitation System Models for Power System Stability Studies", IEEE Std 421.52005 (Revision of IEEE Std 421.5-1992), IEEE April 2006.

[32] ENTSO-E, Final Report Stability Study, "Complementary technical studies for the synchronization of the Turkish power system with the UCTE Power System", 2007. 